\title{
Wavelet p-Leader Non Gaussian Multiscale Expansions for Heart Rate Variability Analysis in Congestive Heart Failure Patients
}

\author{
H. Wendt, Member, IEEE, P. Abry, Fellow, IEEE, K. Kiyono, J. Hayano, E. Watanabe, Y. Yamamoto
}

\begin{abstract}
Objective: Numerous indices were devised for the statistical characterization of temporal dynamics of heart rate variability (HRV) with aim to discriminate between healthy subjects and non healthy patients. Elaborating on the concepts of (multi)fractal and non linear analyses, the present contribution defines and studies formally novel non Gaussian multiscale representations. Methods: A methodological framework for non Gaussian multiscale representations constructed on wavelet $p$-leaders is developed, relying a priori neither on exact scale-free dynamics nor on pre-defined forms of departure from Gaussianity. Its versatility in quantifying the strength and nature of departure from Gaussian is analyzed theoretically and numerically. The ability of the representations to discriminate between healthy subjects and congestive heart failure (CHF) patients, and between survivors and non survivor CHF patients, is assessed on a large cohort of 198 subjects. Results: The analysis leads to conclude that i) scale-free and multifractal dynamics are observed, both for healthy subjects and CHF patients, for time scales shorter than $170 s$; ii) a circadian evolution of multifractal and non Gaussian properties of HRV is evidenced for healthy subjects, but not for CHF patients; iii) non Gaussian multiscale indices possess high discriminative abilities between survivor and non survivor CHF patients, at specific time scales $(\sim 20 s$ and $\sim 85 s)$. Conclusions: The non Gaussian multiscale representations provide evidence for the existence of short-term cascade-type multifractal mechanisms underlying HRV for both healthy and CHF subjects. A circadian evolution of this mechanism is only evidenced for the healthy group, suggesting an alteration of the sympatheticparasympathetic balance for CHF patients. Significance: Results obtained for a large cohort of subjects suggest that the novel non Gaussian indices might robustly quantify crucial information for clinical risk stratification in CHF patients.
\end{abstract}

Index Terms-Multiscale, Non Gaussian, Wavelet Leaders, Heart Rate Variability, Congestive Heart Failure

Work supported by Grant ANR-16-CE33-0020 MultiFracs and CNRS PICS 7260 MATCHA.

H. Wendt is with IRIT-INPT, CNRS, University of Toulouse, France (email: herwig.wendt@irit.fr)

P. Abry is with the Laboratoire de Physique, Université de Lyon, ENS de Lyon, Université Claude Bernard, CNRS, F-69342 Lyon, France (email: patrice.abry@ens-lyon.fr)

K. Kiyono is with the Division of Bioengineering, Graduate School of Engineering Science, Osaka University, Toyonaka, Japan (email: kiyono@bpe.es.osaka-u.ac.jp)

J. Hayano is with the Department of Medical Education, Nagoya City University Graduate School of Medical Sciences, Japan (email: hayano@med.nagoya-cu.ac.jp)

E. Watanabe is with the Department of Cardiology, Fujita Health University School of Medicine, Toyoake, Japan (email: enwatan@fujita-hu.ac.jp)

Y. Yamamoto is with the Educational Physiology Laboratory, Graduate School of Education, University of Tokyo, Japan (email: yamamoto@p.utokyo.ac.jp)

\section{INTRODUCTION}

Heart Rate Variability analysis for risk stratification in Congestion Heart Failure. The fluctuations along time of the Heart Rate, often referred to as Heart Rate Variability (HRV), are known to constitute a valuable information regarding the health status of human beings. It is thus often used to distinguish patients suffering from pathologies from healthy subjects (cf. e.g., [1] and the numerous references therein for a review). Despite HRV monitoring being routinely used in clinical practice, refined analysis of the temporal dynamics underlying HRV have been and remain the subject of numerous and sustained academic research efforts. Indeed, HRV is known to be driven by intertwined interactions between physiological, neurological mechanisms and mechanical constraints, which result in temporal dynamics commonly accepted to be highly irregular and complex, thus requiring advanced statistical signal processing methodologies for analysis.

HRV analysis has notably been used for characterization of Congestive Heart Failure (CHF) of interest here (cf. e.g., [1]-[9]). Important recent advances in therapy for CHF patients were reported in medical studies showing a significant decrease in mortality for patients equipped with implantable cardioverter-defibrillators or undergoing resynchronization therapy. Deciding a priori which patients should benefit from such costly treatments therefore constitutes a major clinical stake. However, this requires to devise reliable a priori risk stratification strategies, which remains a difficult and challenging task that recently received growing academic interest and research efforts (cf., e.g., [2], [3], [10], [11]).

Related works. HRV time series were analyzed in several different ways, naturally starting with classical static (or marginal or first order) descriptive statistics such as the variance of the interarrival times. However, the seminal contribution [12] evidenced that temporal dynamics were key to HRV analysis. This triggered the massive use of spectral analysis (hence second order statistics) for HRV characterization, with indices such as energy in low (LF) and high (HF) frequency bands and spectral slope $\alpha$ [13], [14]. It has since been pointed out repeatedly that achieving classification with sufficient performance and potential use in practice requires to depart from such linear (or first-second order) analyses that implicitly assume Gaussian, stationary short-time correlated models (cf., e.g., [15], [16] and reference therein). Along that line, timefrequency representations and non stationary analysis tools were used, cf. e.g., [17]. Also, several different information 
theoretic quantities were massively used as complexity measures in HRV analysis, including entropy and entropy rate, usually computed using the sample entropy (sampEN) and approximate entropy (apEN) algorithms, respectively, cf., e.g., [4], [5]. More recently, the concepts of fractal and multifractal were also put forward for modeling long-term dependencies (cf. e.g., [2], [6], [7], [18]-[22]). The recently proposed multifractal and scattering analyses also marry nonlinear complexity measures with scale-free dynamics [23], [24]. Alternatively, departures from Gaussianity were also probed as diagnostic tools [3], [8], [9], [25].

Goals, contributions and outline. In this context, elaborating on the concepts of fractal and non linear analyses, the overall goal of the present contribution is to construct flexible non Gaussian multiscale representations that benefit from: i) the use of a large continuum of time scales, yet without requiring a priori exact scale-free dynamics and hence permitting potential departure from multifractal models; ii) the possibility for selecting the (range of) statistical orders for which analysis is to be made, hence clearly departing from the linear, or correlation, or second-order, analysis and its underlying Gaussian assumption. These non Gaussian multiscale representations are embedded within the wavelet $p$-leader formalism, recently defined in [26] and shown to achieve robust estimation of the statistical characteristics of real-world data [27]. They are presented theoretically and illustrated on synthetic time series in Section III. The technique to design non-Gaussian indices generalizes the intuition developed in a specific case in [3], which however relied on the less versatile detrended fluctuation analysis (DFA) multiscale quantities (cf., [27]-[29] for theoretical studies of DFA and comparisons with $p$-leaders).

Moreover, the present analysis intends to investigate the potential benefits of such non Gaussian multiscale representations for studying the differences in HRV between healthy subjects and patients suffering from congestive heart failure $(\mathrm{CHF})$, as well as between survivor and non-survivor $\mathrm{CHF}$ patients. To that end, these tools are applied to a high quality database, described in Section III, consisting of $24 \mathrm{~h} \mathrm{HRV}$ data for $108 \mathrm{CHF}$ patients and 90 healthy subjects collected at Fujita Health University Hospital, Japan.

Results are presented and discussed in Section IV Performance obtained from multifractal and non Gaussian multiscale indices are compared, both for 6-hour day-time period and in terms of evolution along the $24 \mathrm{~h}$. Powers of statistical tests and Kaplan-Meier Survival Curves are used to quantify the significance of differences, with the following main findings: i) multifractal short-term cascade-type temporal dynamics underlying heart rate complexity exist not only for healthy, but also for CHF subjects; ii) for the later group, there is no circadian evolution of multifractality and hence night time deactivation of sympathetic activity; iii) the combined use of non Gaussian multiscale indices is evidenced to possess high discriminative abilities between Non-Survivor (NS) and Survivor (SV) CHF patients at specific time scales and substantially outperforms multifractal and spectral and entropy based indices.
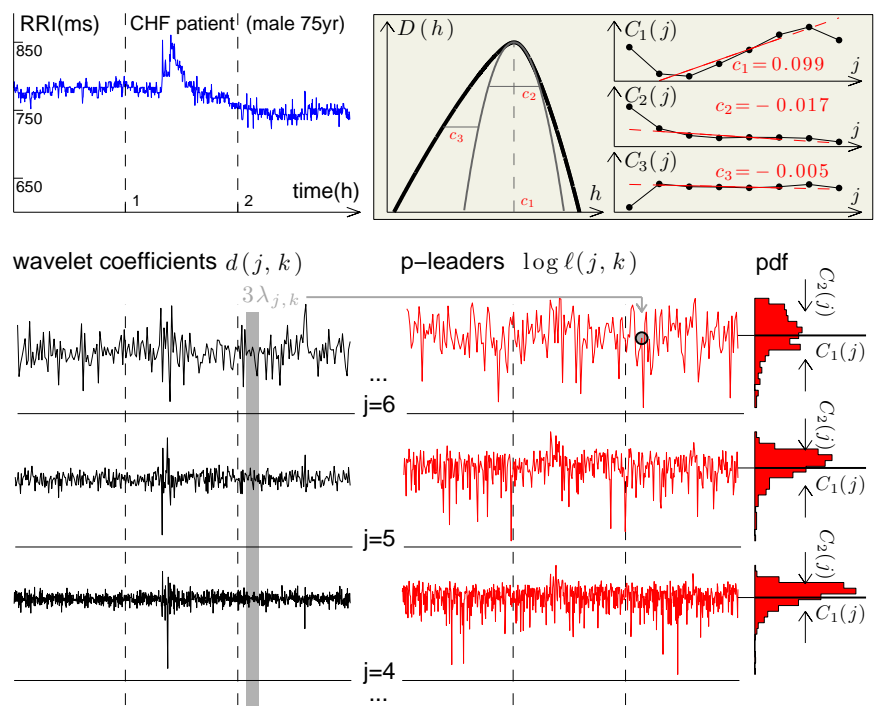

Fig. 1: Illustration of wavelet $p$-leader based multiscale and multifractal analysis.

\section{Methodology}

\section{A. Multiscale quantities}

1) Wavelet coefficients: Let $X(t)$ denote the time series to be analyzed. Given a mother wavelet $\psi$, i.e., a function that is well localized both in time and frequency and characterized by its number of vanishing moments $N_{\psi}$, a strictly positive integer defined as $\forall n=0, \ldots, N_{\psi}-1, \int_{t}^{k} \psi(t) d t \equiv 0$ and $\int_{t}^{N_{\psi}} \psi(t) d t \neq 0$, the $\left(L^{1}\right.$-normalized) discrete wavelet transform (DWT) coefficients $d_{X}(j, k)$ of $X$ are defined by the inner products $d_{X}(j, k)=\left\langle\psi_{j, k} \mid X\right\rangle$, with $\left\{\psi_{j, k}(t)=\right.$ $\left.2^{-j} \psi\left(2^{-j} t-k\right)\right\}_{(j, k) \in \mathbb{N}^{2}}$. We refer the reader to, e.g., [30], for precise definitions and details.

2) Wavelet p-leaders: The $p$-leaders are defined as local $\ell^{p}$ norms of the wavelet coefficients computed from the primitive of $X, Y(t)=\int^{t} X(s) d s$ :

$$
\ell^{(p)}(j, k) \triangleq\left(2^{j} \sum_{\lambda_{j^{\prime}, k^{\prime}} \subset 3 \lambda_{j, k}}\left|d_{Y}\left(j^{\prime}, k^{\prime}\right)\right|^{p} 2^{-j^{\prime}}\right)^{1 / p}
$$

with $\lambda_{j, k}=\left[k 2^{j},(k+1) 2^{j}\right)$ and $3 \lambda_{j, k}=\bigcup_{m\{-1,0,1\}} \lambda_{j, k+m}$. The $p$-leaders, with $p \geq 0$, thus consist of weighted averages of wavelet coefficients existing at all finer scales $2^{j^{\prime}} \leq 2^{j}$ and within a local temporal neighborhood $\lambda_{j^{\prime}, k^{\prime}} \subset 3 \lambda_{j, k}$. For $p$-leaders, estimation benefits from smaller variance, and both positive and negative statistical moments are well defined and numerically stable (unlike, e.g., those of DWT or DFA coefficients), hence permitting the practical assessment of their scale-by-scale distributions, see [26] for precise definitions and details, beyond the scope of this contribution. Following [27], $p=1$ is used in all analyses reported below. The superscript $(p)$ is therefore dropped for notational simplification.

\section{B. Scale-free dynamics and multifractal analysis}

Scale invariance in HRV has already been massively studied, mainly using (second order) spectral analysis and $1 / f$ models, cf., e.g., [15], [16]. The $p$-leaders enable to go beyond such 
a second order analysis of linear properties and permit to quantify multifractal scale invariance.

1) Moments, cumulants and scale invariance: Let $\ell_{s}$ denote a $p$-leader coefficient $\ell(j, k)$ at scale $s=2^{j}$. A standard generating function expansion argument [31], [32] yields a relation for the finite moments $\mathbb{E} \ell_{s}^{q}<\infty$ of $\ell_{s}$ with the cumulants $C_{m}(s)=\mathrm{Cum}_{m} \log \left(\ell_{s}\right)$ of order $m$ of $\log \left(\ell_{s}\right)$

$$
L_{q}(s) \triangleq \log \left(\mathbb{E} \ell_{s}^{q}\right)=\log \mathbb{E} e^{q \log \left(\ell_{s}\right)}=\sum_{m=1}^{\infty} C_{m}(s) \frac{q^{m}}{m !} .
$$

Scale-free temporal dynamics implies a specific form of dependence with respect to scale:

$$
\begin{aligned}
L_{q}(s) & =\kappa_{q}+\zeta(q) \log (s) \\
C_{m}(s) & =c_{m}^{0}+c_{m} \log (s)
\end{aligned}
$$

where (4) follows by substituting (3) in (2) and implies $\zeta(q)=$ $\sum_{m=1}^{\infty} c_{m} \frac{q^{m}}{m !}$, cf., [31]. The scaling exponents $\zeta(q)$ and logcumulants $c_{m}$ provide two alternative ways for quantifying the scale invariance properties of $X$.

2) Scale invariance and multifractal spectrum: The scalefree properties of $X(t)$ are intimately connected with the fluctuations of its point-wise regularity $h(t)$ along $t$, as measured by the multifractal spectrum $D(h)$ : It is theoretically defined as the fractal (Haussdorf) dimension $\operatorname{dim}_{H}$ of the set of instants $t$ where $X(t)$ has identical regularity $h$ and can in practice be computed from either $\zeta(q)$ or $c_{m}$ [33], [34]

$$
\begin{aligned}
D(h) & \triangleq \operatorname{dim}_{H}\{t \mid h(t)=h\} \leq \inf _{q}(1+q h-\zeta(q)) \\
& \approx 1+\frac{c_{2}}{2 !}\left(\frac{h-c_{1}}{c_{2}}\right)^{2}+\frac{-c_{3}}{3 !}\left(\frac{h-c_{1}}{c_{2}}\right)^{3}+\ldots
\end{aligned}
$$

When based on $p$-leaders, the regularity at time $t$ is measured by the p-exponent $h(t)$, a generalization of the usual Hölder exponent, cf., [26], [27]. Expressions (3)6] highlight the strong link between $D(h)$ and the scale invariance properties of $X$. In particular, $c_{1}$ quantifies the average local regularity of $X ; c_{m}$, $m \geq 2$, relate to the multifractal properties of data, notably $c_{2}$ quantifies the degree of fluctuation of local regularity, and $c_{3}$ the asymmetry of these fluctuations about $c_{1}$.

Finally, (3 6) permit the robust assessment of $\zeta(q), c_{m}$ and $D(h)$ for discrete data $X$ : by replacing theoretical moments $\mathbb{E}(\cdot)^{q}$ or cumulants $\mathrm{Cum}_{m}$ in (3) or (4) with sample moments or cumulants, estimates of $\zeta(q), c_{m}$ and $D(h)$ can be obtained from linear regression of $L_{q}(s)$ or $C_{m}(s)$ for a range of scales $\log _{2}(s)=j \in\left[j_{1}, j_{2}\right]$, cf., [32], [34]. The $p$-leader multiscale and multifractal analysis of a HRV time series is schematically represented in Fig. 11 Other multiscale quantities have been previously proposed, notably also based on (discrete) wavelet transforms [32], [34], [35] (cf., [36] for a review).

3) Cumulants $C_{m}(s)$ and departures from Gaussianity: The cumulants $C_{m}(s)$ underlying the multifractal formalism, cf., (2), provide a description of the distribution of $\log \left(\ell_{s}\right)$ : e.g., $C_{1}, C_{2}, C_{3}$ and $C_{4}$ correspond to the mean, variance, skewness and kurtosis, etc. This can be illustrated using three prominent Gaussian and non Gaussian model processes for scale-free dynamics: The seminal fractional Brownian motion (fBm) [37], which is Gaussian, self-similar and satisfies $C_{m}(s)=$ const for $m \geq 2$; Multifractal random walk (MRW), which is non Gaussian of a special type of departure from Gaussian referred to here as log-normal, cf., [38], [39], specifically, $C_{2}(s) \neq$ const while $C_{m}(s)=$ const for $m \geq 3$; Compound Poisson cascade (CPC), which is another multifractal process with a more general form of non Gaussianity than MRW, i.e., $C_{m}(s) \neq$ const $\forall m \geq 2$, cf. [40] (process parameters are chosen to yield identical $C_{1}(s)$ for all processes and identical $C_{2}(s)$ for MRW and CPC). Fig. 2 compares $C_{m}(s)$ for these processes: $C_{1}(s)$ only captures the linear, Gaussian (mean and variance/covariance) properties and thus does not permit to distinguish the three processes; $C_{2}(s)$ differs between Gaussian fBm and non Gaussian MRW \& CPC but collapses on one curve for the latter two processes; $C_{3}(s)$ is essentially identical for Gaussian $\mathrm{fBm}$ and non Gaussian MRW as both processes have symmetrical distributions, yet differs for CPC which is designed to have a non symmetrical distribution. While theoretically different for all three processes, $C_{4}(s)$ are observed to fall within error bars. Therefore, beyond permitting a valuable descriptions of scale-free and multifractal dynamics, the $C_{m}(s)$ also relate to departures from Gaussian.

4) Limitations: Scale-free and multifractal analyses have already been massively employed to analyze HRV both in adults [6], [36] and fetuses [23], [34]. Nevertheless, the a priori assumption of exact scale free dynamics implies a special form of scale dependence as in (34), and the sole use of scaling exponents $\zeta(q)$ or $c_{m}$ can be limiting in applications. In addition, non Gaussian properties over scales $s$ were reported to be useful for characterizing HRV, cf., [3], [8], [9]. Cumulants $C_{m}(s)$ quantify non Gaussianity of the $\log \left(\ell_{s}\right)$, yet there is no reason a priori that one single cumulant of a specific order should be relevant for a particular application. Further, $C_{m}(s)$ of high order $m$ may be difficult to estimate accurately in practice. This motivates the definition of novel, versatile non Gaussian multiscale representations.

\section{Non Gaussian multiscale representations}

1) Definition and key properties: Inspired by the generalized moments approach introduced in turbulence literature [41] and motivated by the non Gaussian index studied in [3], we propose here to define non Gaussian representations as linear combinations of cumulants. Specifically, we define a non Gaussian multiscale expansion of $X$, with $P \in \mathbb{N}^{+}$and $\boldsymbol{q}=\left(q_{1}, \ldots, q_{2 P}\right), q_{i} \neq 0$ and $q_{i} \neq q_{j}$, as:

$$
\mathbf{L}_{\boldsymbol{q}}^{(2 P)}(s) \triangleq \log \left(\prod_{i=1}^{P} \frac{\left(\mathbb{E} \ell_{s}^{q_{2 i-1}}\right)^{\frac{1}{q_{2 i-1}}}}{\left(\mathbb{E} \ell_{s}^{q_{2 i}}\right)^{\frac{1}{q_{2 i}}}}\right)
$$

Making use of (2), it is straighforward to show that $\mathbf{L}_{\boldsymbol{q}}^{(2 P)}(s)$ equals an infinite weighted sum of the cumulants $C_{m}(s)$

$$
\begin{aligned}
\mathbf{L}_{\boldsymbol{q}}^{(2 P)}(s) & =\sum_{i=1}^{P} \frac{L_{s}\left(q_{2 i-1}\right)}{q_{2 i-1}}-\frac{L_{s}\left(q_{2 i}\right)}{q_{2 i}} \\
& =\sum_{m=2}^{\infty} C_{m}(s) \frac{\sum_{i=1}^{P} q_{2 i-1}^{m-1}-q_{2 i}^{m-1}}{m !} .
\end{aligned}
$$

Property 0: The evolution across scales $s$ of $\mathbf{L}_{\boldsymbol{q}}^{(2 P)}(s)$ is an indicator for the non Gaussian properties of $X$ : Indeed, 

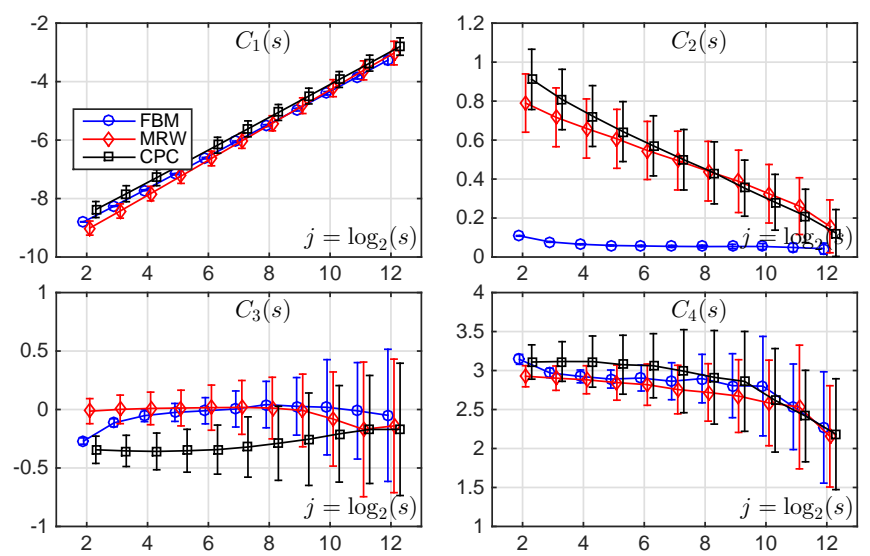

Fig. 2: Cumulants $C_{m}(s)$ for Gaussian self-similar fBm (blue solid) and non Gaussian multifractal MRW and CPC (red and black solid lines, respectively).
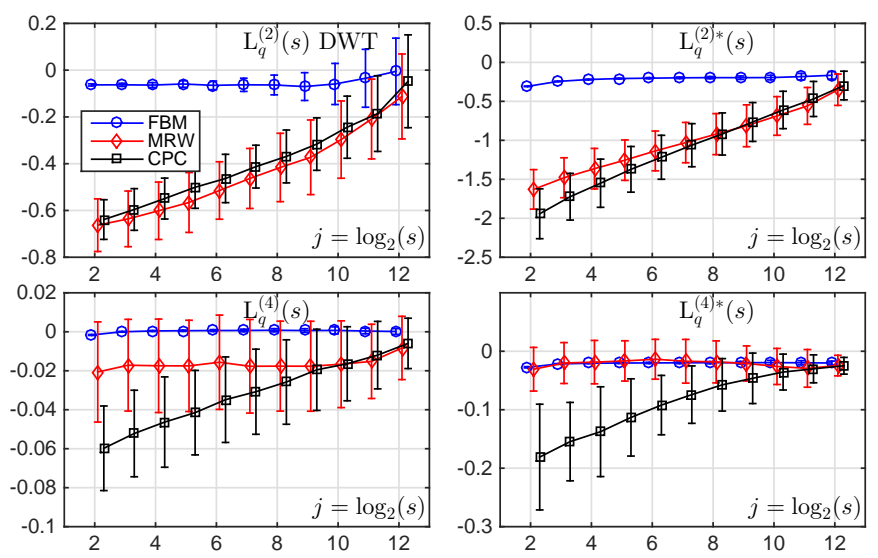

Fig. 3: Non Gaussian indices $L_{s}^{(2)}(\boldsymbol{q}), L_{s}^{(4)}(\boldsymbol{q})$ for Gaussian self-similar fBm (blue solid) and non Gaussian multifractal MRW and CPC (red and black solid lines, respectively).

for Gaussian data, it is straightforward to show that $\mathbf{L}_{\boldsymbol{q}}^{(2 P)}(s)$ equals a constant, whose value depends only on the choice of multiresolution quantity (here, $p$-leaders). Fig. 3 illustrates this for Gaussian fBm (blue lines): $\forall P, \forall \boldsymbol{q}, \mathbf{L}_{\boldsymbol{q}}^{(2 P)}(s)=$ const. Property 1: $\mathbf{L}_{\boldsymbol{q}}^{(2 P)}(s)$ does not depend on the value of $C_{1}(s)$ because all $q_{2 i}^{m-1}-q_{2 i+1}^{m-1}$ equal zero for $m=1$. Therefore, $\forall P, \forall \boldsymbol{q}, \mathbf{L}_{\boldsymbol{q}}^{(2 P)}(s)$ solely measures the nonlinear properties or departures from Gaussianity for $X$ (cf., Fig. 3 ).

Property 2: The relative weighting of the cumulants $C_{m}(s)$ in the expansion $\mathbf{L}_{\boldsymbol{q}}^{(2 P)}(s)$ can be tuned and customized by choosing $P$ and $\boldsymbol{q}$ appropriately. This versatility can be used to quantify, e.g., the strength of deviation from Gaussianity, or to finely characterize the nature of this departure.

Property 3: $\mathbf{L}_{\boldsymbol{q}}^{(2 P)}(s)$ permits to probe cumulants of high order, that would turn difficult to estimate, using only moments $L_{q}(s)=\log \left(\mathbb{E} \ell_{s}^{q}\right)$ of low orders $q$.

2) Versatility: The possible uses of this non Gaussian multiscale expansion are now illustrated on several examples. Historical example: In [3], a first non Gaussian index was proposed and used for the multiscale assessment of the strength of deviation from Gaussian of HRV. It relied on detrended increments (i.e., quantities reminiscent of DWT coefficients) instead of $\ell_{s}$, and on the specific choice $P=1$, $\boldsymbol{q}=(0.25,2)$. Fig. 4 (top left) shows that it mostly probes $C_{2}(s)$ and $C_{3}(s)$, while Fig. 3 (1st row, left column) shows that its evolution across scales effectively discriminates Gaussian $\mathrm{fBm}$ (blue line) from non Gaussian MRW and CPC (red and black lines, respectively). Yet, it does not permit the discrimination of the latter two non Gaussian processes.

Removing $C_{2}(s)$ : We can refine the non Gaussian multiscale assessment and cancel any cumulant of order $m=M \geq 2$ in (8) by setting $P \geq 2$ and $\boldsymbol{q}$ such that $\sum_{i=1}^{P} q_{2 i-1}^{m-1}-q_{2 i}^{m-1}=$ 0 , subject to $q_{2 i-1} \neq q_{2 j}, i, j=1, \ldots, P$. The specific choice $P=2, \boldsymbol{q}=(u, u+r, v, v-r), r \neq\{0,-u, v, v-u$, leads to removal of $C_{2}(s)$ in $\mathbf{L}_{\boldsymbol{q}}^{(4)}(s)$, as illustrated in Fig. 4 (bottom left). This enables us to discriminate non Gaussian of log-normal nature from other types of non Gaussian. Fig. 3 (bottom left) shows that this permits distinguishing the lognormal type MRW (red line) from the more general departure from Gaussian found in CPC (black line).

Removing odd cumulants $C_{2 m+1}(s)$ : Moreover, the use of $p$-leader coefficients $\ell_{s}$ enables us to use moments of negative order $q$ in the construction of non Gaussian expansion, leading to additional desirable properties. Specifically, the choice $q_{2 i-1}=-q_{2 i}, q_{2 i-1} \neq q_{2 j}, i, j=1, \ldots, P$, for $P \geq 1$ (i.e., $\boldsymbol{q}=(q,-q)$ for $P=1)$ removes all $C_{m}(s)$ of odd order $m$ from (8), as illustrated in Fig. 4 (top right). It thus permits to focus mainly on non Gaussian properties of symmetric nature. What is more, Fig. 3 (1st row, right column) shows that the negative-moment-based index leads to smaller (relative to variation with scale) error bars and facilitates distinguishing Gaussian (blue line) from non Gaussian (red and black lines). Removing even cumulants $C_{2 m}(s)$ : All cumulants of even order can be cancelled from $\mathbf{L}_{\boldsymbol{q}}^{(2 P)}(s)$ by setting $P \geq 2$ and $q_{i}=-q_{i+P}, \quad i=1, \ldots, P$ subject to $q_{2 i-1} \neq$ $q_{2 j}, \quad i, j=1, \ldots, P$. This choice hence permits to jointly quantify the asymmetry properties; for $P=2$, this reads $\boldsymbol{q}=\left(q_{1}, q_{2},-q_{1},-q_{2}\right), q_{1} \neq q_{2}$ and is illustrated in Fig. 4 (bottom right). Fig. 3 (bottom right) shows that this reduces relative error bar size and increases the contrast between lognormal type (red line) and general (black line) non Gaussian. Remark 1. The proposed expressions of form (8) permit to jointly evaluate higher-order cumulants $C_{m}(s)$ by using moments of smaller order $q_{i}<m$. As an example, for the weights in Fig. 4 (bottom row), $\mathbf{L}_{q}^{(4)}$ is essentially a combination of the higher-order cumulants $C_{3}$ to $C_{7}$, but is computed with moments of order $|q| \leq 2.5$.

Remark 2. With the assumption of scale invariance for $X$ (i.e., (3) and (4)), (8) is of the form $\mathbf{L}_{\boldsymbol{q}}^{(2 P)}(s)=K_{\boldsymbol{q}}+$ $\log (s) \sum_{m=2}^{\infty} c_{m} \frac{\sum_{i=1}^{P} q_{2 i-1}^{m-1}-q_{2 i}^{m-1}}{m !}$ where we have gathered the terms independent of scale $s$ induced by $\log \kappa_{q_{i}}$ in the constant $K_{q}$. The weights in this sum are identical to those in 8 .

\section{Multiscale HRV Characteristics}

During a period of two years (2000 and 2001), a cohort of 61 male and 47 female CHF patients, with age ranging from 21 to 92 (average $66.1 \pm 14.8$ ) years was monitored at Fujita Health University Hospital, Japan, out of which 39 (36.1\%) 

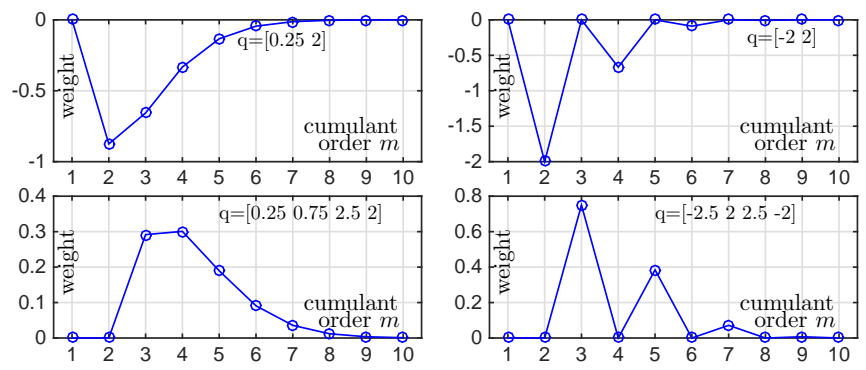

Fig. 4: Weights for the cumulants in $\mathbf{L}^{(2)}(\boldsymbol{q})$ with $\boldsymbol{q}=$ $(0.25,2)$ (top left) and $\boldsymbol{q}=(-2,2)$ (top right), and $\mathbf{L}^{(4)}(\boldsymbol{q})$ with $\boldsymbol{q}=(0.25,0.75,2.5,2)$ (bottom left), and $\boldsymbol{q}=$ $(-2.5,2,2.5,-2)$ (bottom right). Choices reported in the right column focus on either even (top) or odd (bottom) cumulants, those in the bottom row exclude $C_{2}$ from the analysis.

\begin{tabular}{|c|c|c|}
\hline notation & moments $\boldsymbol{q}$ & cumulants $C_{m}$ in $(8)$ and interpretation \\
\hline $\mathbf{L}_{\boldsymbol{q}}^{(2)}(s)$ & $(0.25,2)$ & $\begin{array}{l}m \geq 2 \\
\text { any deviation from Gaussian }\end{array}$ \\
\hline $\mathbf{L}_{\boldsymbol{q}}^{(2) *}(s)$ & $(-2,2)$ & $\begin{array}{l}\text { even order } m=2,4, \cdots \\
\text { mainly symmetric properties of non Gaussian }\end{array}$ \\
\hline $\mathbf{L}_{\boldsymbol{q}}^{(4)}(s)$ & $(0.25,0.75,2.5,2)$ & $\begin{array}{l}m \geq 3 \\
\text { non log-normal nature of non Gaussian }\end{array}$ \\
\hline $\mathbf{L}_{\boldsymbol{q}}^{(4) *}(s)$ & $(-2.5,2,2.5,-2)$ & $\begin{array}{l}m \geq 3 \text { of odd order: } m=3,5, \cdots \\
\text { asymmetry of non Gaussian }\end{array}$ \\
\hline
\end{tabular}

TABLE I: Non Gaussian multiscale indices $\mathbf{L}_{\boldsymbol{q}}^{(2 P)}(s)$ used in the present work.

died within the follow-up period of $33 \pm 17$ months (range 1-59 months). Medication status before hospital discharge did not significantly differ between survivor (SV) and non-survivor (NS) patients. (cf. [3] for further details).

Before discharge from the Hospital, a 24-hour Holter ECG recording was collected for each single patient. $\mathrm{R}$ peaks were extracted from the HRV recordings and thoroughly reviewed for detection error correction and outlier removal. When atrial or ventricular premature complexes occurred, they were handled by median interpolation using two successive beats. Moreover, it has been checked that no sustained tachyarrhythmias were present in the recordings. The RR inter-arrival time values extracted from the HRV recordings are resampled at $4 \mathrm{~Hz}$ using cubic splines and analyzed as a time series, denoted as $X \equiv\left\{x_{n}, n=1, \ldots, N\right\}$. An example of a 3 hours block of one raw time series (male, CHF, 75yr, not resampled) and a

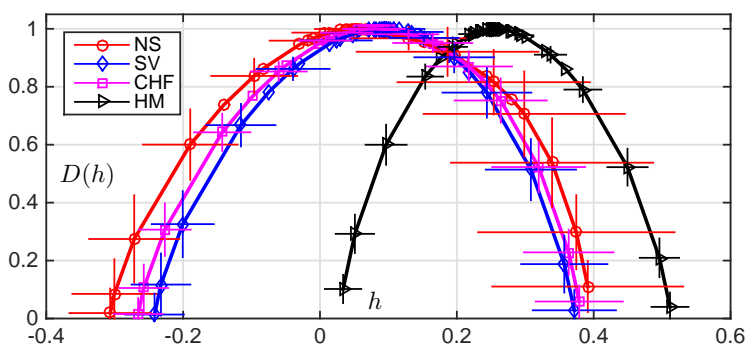

Fig. 5: Multifractal spectra $D(h)$ (averages and 95\% error bars) for scales $(5.3 s, 170.7 s)$ for daytime HRV.
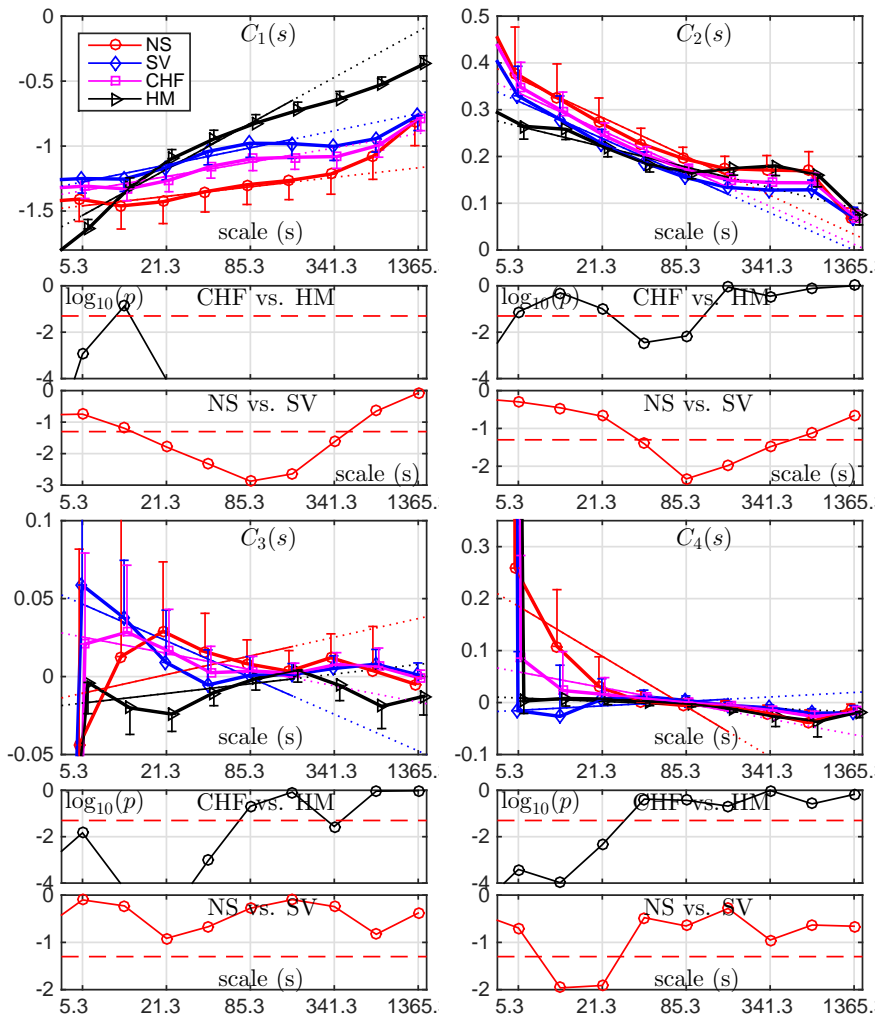

Fig. 6: Cumulants $C_{m}(s)$ as a function of scale $s$ (in seconds; top subplots; averages and $95 \%$ error bars), and WRS p-values for CHF vs. HM and NS vs. SV (bottom subplots) for daytime HRV.

sketch of its multifractal and multiscale analyses are plotted in Fig. 1. For comparison, we use an age-matched healthy (HM) control group of 90 subjects. The study was approved by the ethics committee of Fujita Health University and conformed to the principles outlined in the Declaration of Helsinki. All patients provided written informed consent.

\section{RESUlTS AND Discussion}

\section{A. Estimation setting and statistical testing}

The multifractal and multiscale analyses are performed using a Daubechies wavelet with $N_{\psi}=3$ vanishing moments (the use of a different wavelet leads to similar conclusions). Linear regressions for multifractal coefficients $c_{m}$ are computed for octaves $j_{1}=4 \leq j \leq j_{2}=9$, corresponding to scales $5.3 \mathrm{~s}$ to $170.7 \mathrm{~s}$, cf., Sec. IV-B1, the multiscale indices $\mathbf{L}_{\boldsymbol{q}}^{(2 P)}(s)$ are defined in Tab. I. Two different analyses will be performed: i) analysis of the daytime HRV for a 6 hour long block; ii) analysis of the evolution of HRV properties over $24 \mathrm{~h}$ for $2 \mathrm{~h}$ long blocks with $1 \mathrm{~h}$ overlap.

To assess differences between groups, pairwise Wilcoxon rank-sum (WRS) test p-values are computed. Between group differences for NS vs. SV are further evaluated using KaplanMeier cumulative survival curves (KMCS) and the MantelHaenszel (MH) log rank test.

\section{B. Multifractal properties of $H R V$}




\begin{tabular}{|c|c|c|r|c|}
\hline Estimates & $c_{1}$ & $c_{2}$ & $c_{3}$ & $c_{4}$ \\
\hline HM & 0.255 & -0.032 & 0.005 & -0.005 \\
\hline CHF & 0.079 & -0.058 & -0.008 & -0.022 \\
\hline NS & 0.053 & -0.060 & 0.009 & -0.070 \\
\hline SV & 0.095 & -0.057 & -0.017 & 0.006 \\
\hline \hline WRS p-value & $c_{1}$ & $c_{2}$ & $c_{3}$ & $c_{4}$ \\
\hline CHF vs. HM & $<1 e-8$ & 0.276 & $3.0 e-4$ & 0.111 \\
\hline NS vs. SV & 0.156 & 0.720 & 0.932 & 0.068 \\
\hline
\end{tabular}

TABLE II: Average multifractal coefficients $c_{m}$ for scales $(5.3 s, 170.7 s)$ (top) and pairwise WRS p-values (bottom).

\begin{tabular}{|c|c|r|r|r|r|r|}
\hline Estimates & LF & \multicolumn{1}{c|}{ HF } & LF/HF & $\alpha_{P S D}$ & sampEN & apEN \\
\hline HM & 0.057 & 0.028 & 3.001 & 2.314 & 0.267 & 0.324 \\
\hline CHF & 0.064 & 0.160 & 0.713 & 1.362 & 0.431 & 0.600 \\
\hline NS & 0.064 & 0.132 & 0.692 & 1.476 & 0.378 & 0.538 \\
\hline SV & 0.064 & 0.176 & 0.725 & 1.300 & 0.463 & 0.637 \\
\hline \hline WRS p-value & $L F$ & $H F$ & $L F / H F$ & $\alpha_{P S D}$ & sampEN & apEN \\
\hline CHF vs. HM & 0.435 & $<1 e-8$ & $<1 e-8$ & $<1 e-8$ & $<1 e-8$ & $<1 e-8$ \\
\hline NS vs. SV & 0.406 & 0.173 & 0.927 & 0.327 & 0.062 & 0.127 \\
\hline
\end{tabular}

TABLE III: Top: spectral (col. 2-5, $(\mathrm{V} / \mathrm{ms})^{2}$ ) and entropy (col. 6-7) features; bottom: pairwise WRS p-values.

1) Scale invariance and multifractality in daytime $H R V$ : Fig. 6 (top row) plots the functions $C_{m}(s), m=1,2,3,4$ (from left to right; averages and 95\% error bars) for NS, SV, CHF and HM, for the 6 hour long blocks recorded between $17 \mathrm{~h} 00$ and $23 \mathrm{~h} 00$. All groups exhibit scale-free dynamics (i.e., power laws) for scales ranging from about 5 s to 170 s, that is, in scales corresponding to LF and VLF of traditional spectral analysis [1]. While scale-free dynamics have been reported for healthy subjects in [8], [25], [42], our findings suggest that such scale-free dynamics are also observed for CHF patients, cf., Fig. 6

The manifestation of scale-free dynamics for all groups justifies the calculation and comparison of the multifractal spectra $D(h)$, plotted in Fig. 5 The corresponding average multifractal coefficients $c_{m}, m=1,2,3,4$, are given in Tab. III. Both CHF and HM are characterized by nicely shaped average multifractal spectra, indicating well-behaved concave scaling functions $\zeta(q)$ as predicted by multifractal theory [40], [43] and an excellent fit for multifractal models for all groups, including CHF. Together, these findings permit to postulate that there is a multifractal mechanism underlying heart rate complexity, not only for healthy subjects as reported in [8], [25], [42] but also for CHF subjects. This suggests cascade-type dynamics in HRV data, both for healthy and CHF subjects, at time scales below 3 minutes.

Further, the mode $c_{1}$ of the multifractal spectrum, quantifying the average degree of regularity of HRV, is found to be larger for HM than for CHF. For the width of $D(h)$, related to $\left|c_{2}\right|$ and quantifying the range of fluctuations of the regularity of HRV along time, the converse holds: it is smaller for HM than for CHF Together, these two observations indicate that $\mathrm{HRV}$ of CHF is characterized by overall more irregular, erratic time evolution, than that of $\mathrm{HM}$, both globally (smaller $c_{1}$ ) and locally (more multifractility, larger $\left|c_{2}\right|$ ), and corroborates similar findings on the self-similarity exponent $H$ [9. Table 5]. Note that similar observations hold for SV and NS (smaller $c_{1}$ and larger $\left|c_{2}\right|$ for NS than SV), but differences are not
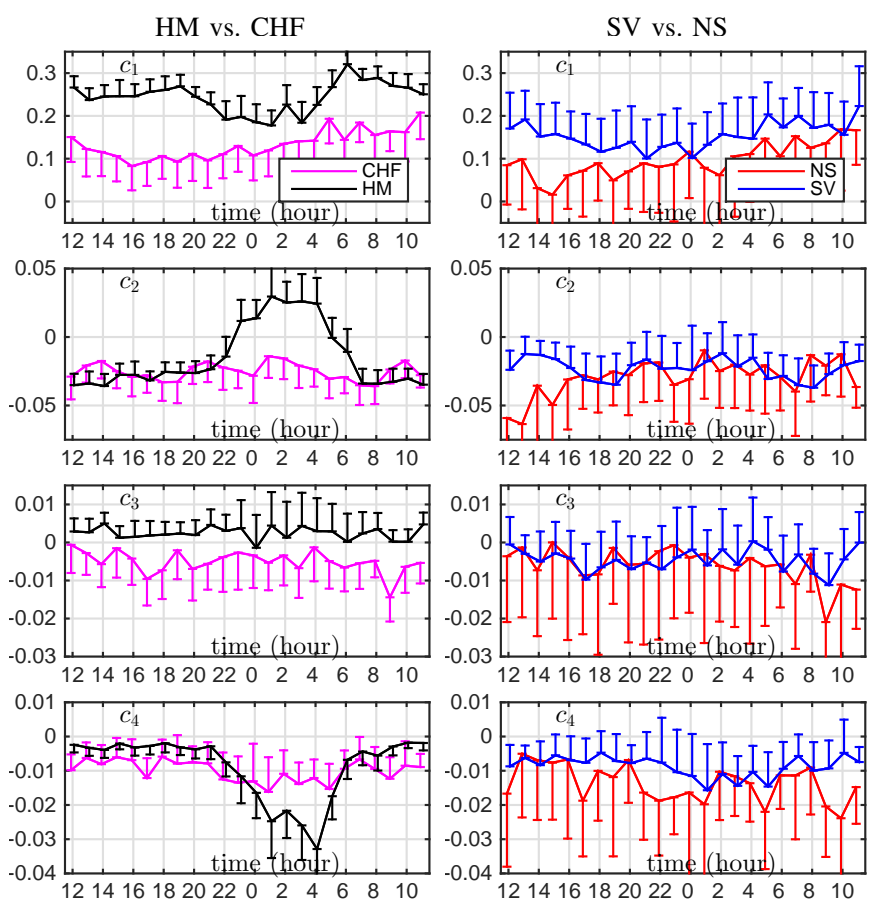

Fig. 7: Estimates for for $c_{m}, m=1,2,3,4$ (top to bottom row; median and $2.91 \cdot \mathrm{mad} / \sqrt{N}$ ) obtained for $2 \mathrm{~h}$ long windows with $1 \mathrm{~h}$ overlap, plotted as a function of window central time for HM \& CHF (left column) and NS \& SV (right column).

significant, as discussed next.

2) Discriminative power of multifractal properties of HRV: WRS p-values between the groups HM, CHF and NS, SV for $c_{m}, m=1,2,3,4$, reported in Tab. II bottom rows), assess their independent discriminative power: First, for HM vs. CHF, $c_{1}$ and $c_{3}$ have extremely high $(p<1 e-12)$ and high $(p<0.001)$ significance, respectively, while $c_{2}$ and $c_{4}$ are not significant. Second, for NS vs. SV, none of the multifractal coefficients are significant $(p>0.05)$. We conclude that the differences in multifractal dynamics of HRV are discriminative for healthy vs. CHF, but not for NS vs. SV subjects, excluding their use as indicators of mortality risk stratification in CHF.

For comparison, Tab. III shows average estimates and pvalues for the spectral and information theoretic analysis methods described in Sec. Ir energy in the low (LF) and high (HF) frequency band, LF/HF ratio and spectral slope $\alpha_{P S D}$, sampEN and apEN (computed using standard parameters, embedding dimension $m=2$ and ball radius $r=0.2 \times$ data standard deviation). The results lead to similar conclusions: Differences are significant for HM vs. CHF ( $p<1 e-8$, except for LF) but not discriminative for NS vs. SV subjects.

3) Circadian evolution of multifractal properties: To assess the evolution of the multifractal properties of HRV along 24 hours of a day, estimates of $c_{m}, m=1,2,3,4$ (from top to bottom) obtained for 2 hour long blocks with one hour overlap, for HM, CHF and NS, SV (left and right column) are plotted as a function of the block center in Fig. 77. For the control group HM, there is a clear day-night evolution of multifractal parameters, characterized by a significant decrease for the magnitudes of $c_{1}$ and $c_{2}$ during sleep. This decrease 

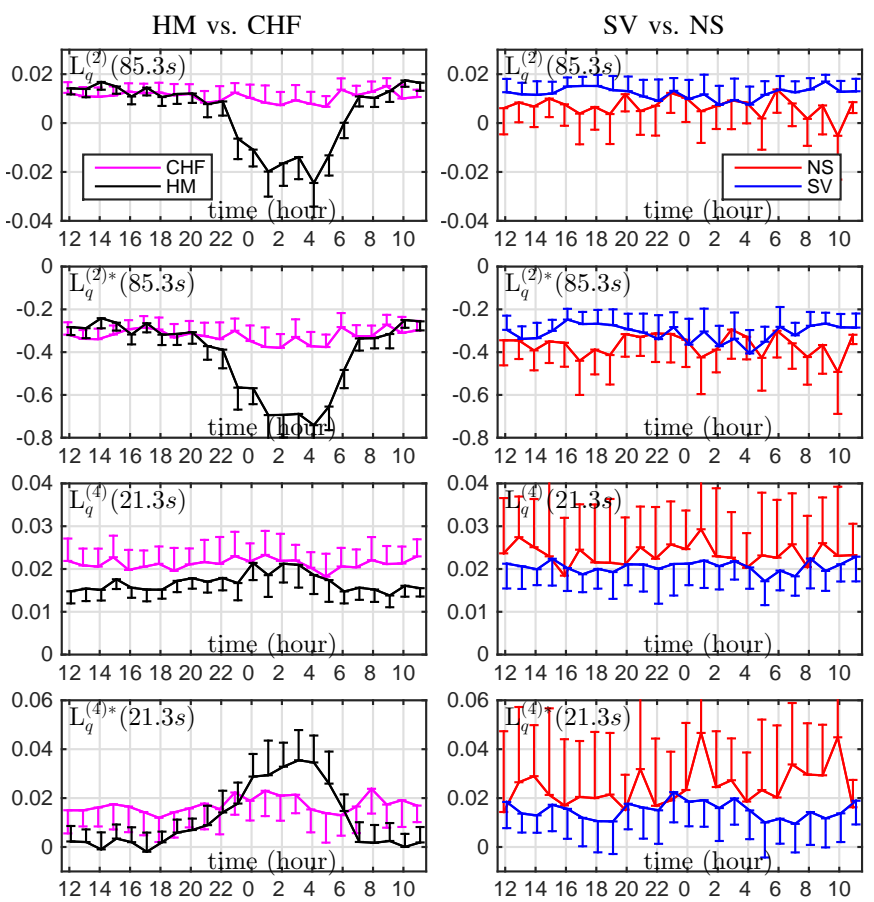

Fig. 8: Estimates for for $\mathbf{L}_{\boldsymbol{q}}^{(2)}(s)$ and $\mathbf{L}_{\boldsymbol{q}}^{(4)}(s)$ (median and $2.91 \cdot \mathrm{mad} / \sqrt{N})$ obtained for $2 \mathrm{~h}$ long windows with $1 \mathrm{~h}$ overlap, plotted as a function of window central time for HM \& CHF (left column) and NS \& SV (right column).

is caused by the deactivation of sympathetic predominance during sleep [44] Interestingly, there also appears to be a significant increase of the magnitude of $c_{4}$ during sleep, which has not been reported before. During sleep, because of the intermittent changes of sleep stages, non Gaussian behavior having heavy tails was observed (see [42]). These intermittent changes of sleep stages may result in the negative $c_{4}$.

For CHF patients, a comparable circadian modulation of the multifractal properties of HRV cannot be observed. At best, a weak difference is found between early morning and evening for the values of $c_{1}$ for SV. This suggests that for CHF patients, the sympathetic-parasympathetic balance does not follow the circadian cycle of healthy subjects. In particular, it lacks a daily period with reduced or without sympathetic activity.

\section{Multiscale properties of HRV}

1) Cumulants $C_{m}(s)$ : The cumulants $C_{m}(s)$, plotted in Fig. 6(top rows), together with WRS p-values for HM vs. CHF and NS vs. SV, as a function of each independent scale $s$ (bottom rows) show that: i) The cumulant $C_{1}(s)$ strongly differs between HM and CHF and between NS and SV, for a large range of time scales from 20 to 350 seconds. ii) The cumulants $C_{2}(s)$ and $C_{4}(s)$ also have significant p-values (yet larger than $C_{1}$ ) for both pairs of groups. iii) There are no significant difference between NS and SV for the third cumulant $C_{3}(s)$. iv) Interestingly, the scales yielding smallest WRS p-values are different for $\left\{C_{1}, C_{2}\right\}$ and $\left\{C_{3}, C_{4}\right\}$ and are identified as $85.3 \mathrm{~s}$ and $21.3 \mathrm{~s}$, respectively.

2) Non Gaussian indices $\mathbf{L}_{q}^{(2 P)}(s)$ : Fig. 9 plots the wavelet $p$-leader based multiscale non Gaussian indices $\mathbf{L}_{\boldsymbol{q}}^{(2 P)}(s)$. A
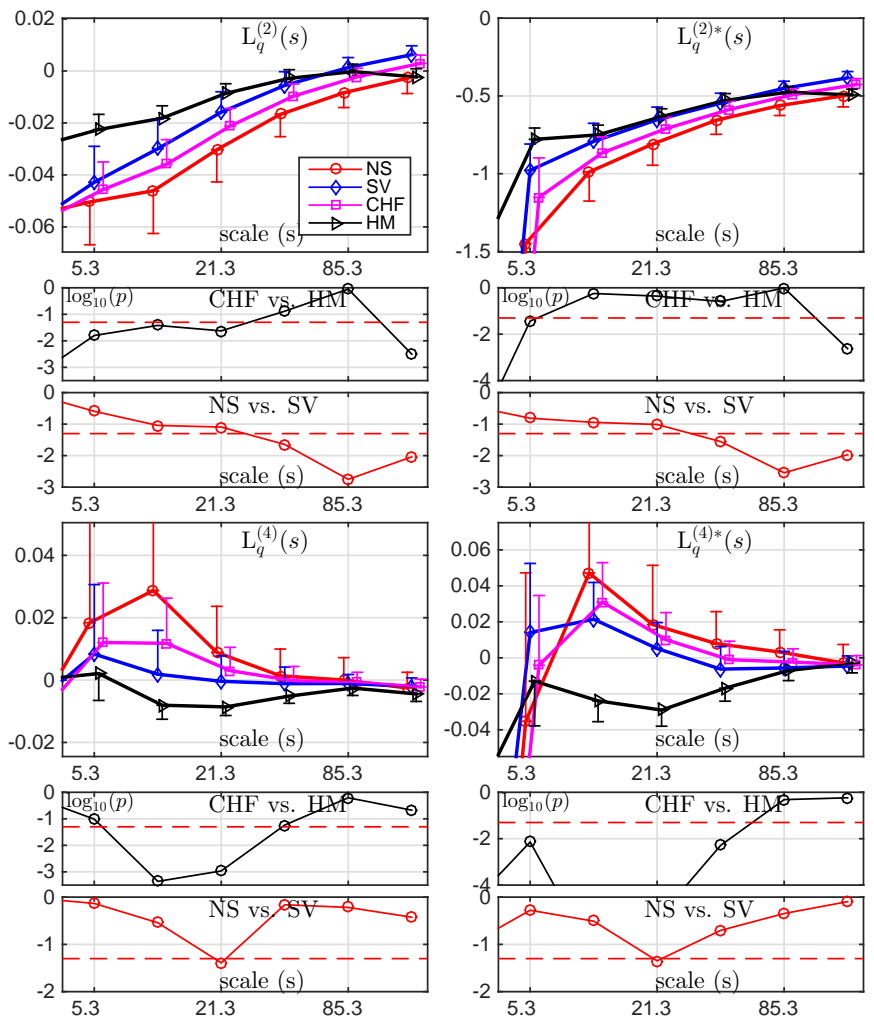

Fig. 9: non Gaussian indices $\mathbf{L}_{\boldsymbol{q}}^{(2)}(s)$ and $\mathbf{L}_{\boldsymbol{q}}^{(4)}(s)$ as a function of scale $s$ (in seconds; averages and 95\% error bars, top rows), and WRS p-values for CHF vs. HM and NS vs. SV (bottom rows) for daytime HRV.

first important observation is that the non Gaussian indices $\mathbf{L}_{q}^{(2)}(s)$ and $\mathbf{L}_{q}^{(2) *}(s)$ display a significant evolution across scales, confirming that HRV has non Gaussian multiscale statistics. Yet, they tend to a constant value - and hence the distribution of $\ell_{s}$ converges to Gaussian - for large scales $\geq 170 \mathrm{~s}$. Such a behavior is inherent to and a key characteristic of multifractal cascades, cf., e.g., [31]. It thus further strengthens our conclusions on the cascade-type multifractal nature of heart rate dynamics for both healthy and CHF subjects, cf., Section IV-B1.

The magnitude of $\mathbf{L}_{q}^{(2)}$ is an indicator for the degree of non Gaussianity and is found to be larger for CHF than for $\mathrm{HM}$, and larger for NS than for SV: Thus, CHF is stronger non Gaussian than HM, and NS stronger than SV. These differences are found to be significant for HM vs. CHF at fine scales, and for NS vs. SV in the vicinity of scale $85.3 \mathrm{~s}$. The observation of significantly more non Gaussian distributions for HRV of NS than SV corroborates findings reported in [3].

Indications on the nature of the departure from Gaussian for the multiscale distributions of heart rate dynamics are obtained from $\mathbf{L}_{\boldsymbol{q}}^{(4)}(s)$ and $\mathbf{L}_{\boldsymbol{q}}^{(4) *}(s)$. They systematically take on nonzero values and thus suggest that the departures from Gaussian are not of log-normal type. Moreover, these observations are consistent between $\mathbf{L}_{\boldsymbol{q}}^{(4)}(s)$ and $\mathbf{L}_{\boldsymbol{q}}^{(4) *}(s)$. This suggests that the difference in multiscale statistics between NS and SV is to large extent driven by asymmetry because the index $\mathbf{L}_{\boldsymbol{q}}^{(4) *}(s)$ does not depend on cumulants of odd order. These deviations 
from simple log-normal type non Gaussian are stronger for $\mathrm{CHF}$ than for HM, and stronger for NS than for SV. They are significant for CHF vs. HM, and to a lesser extent also for NS vs. SV, at scales in the vicinity of $21.3 s$.

To conclude, differences in the amount and in the nature of the deviations from Gaussian are found significant between groups. Interestingly, the most significant time scales do not coincide for non Gaussian amount (85.3s for $\mathbf{L}_{\boldsymbol{q}}^{(2)}$ and $\mathbf{L}_{\boldsymbol{q}}^{(2 *)}$ ) and nature (21.3s for $\mathbf{L}_{\boldsymbol{q}}^{(4)}$ and $\mathbf{L}_{\boldsymbol{q}}^{(4)}$ ). This is in agreement with earlier observations on $C_{m}$ (since $\mathbf{L}_{\boldsymbol{q}}^{(2)}$ mostly depend on $C_{2}$, and $\mathbf{L}_{\boldsymbol{q}}^{(4)}$ only on $C_{m}, m \geq 3$ ).

3) Circadian evolution of non Gaussian indices: To assess the evolution of the multiscale non Gaussian properties of HRV along 24 hours of a day, estimates of $\mathbf{L}_{\boldsymbol{q}}^{(2 P)}(s)$ obtained for 2 hour long blocks with one hour overlap, for the scales identified as significant in the paragraph section, for HM, $\mathrm{CHF}$ and NS, SV (left and right column) are plotted as a function of the block center in Fig. 8. For the control group HM, there is a clear and distinct day-night evolution for all non Gaussian indices $\mathbf{L}_{\boldsymbol{q}}$. Specifically, the values for $\mathbf{L}_{\boldsymbol{q}}^{(2)}(85.3 s)$ and $\mathbf{L}_{\boldsymbol{q}}^{(2) *}(85.3 s)$ decrease during night time, while $\mathbf{L}_{\boldsymbol{q}}^{(4)}(21.3 s)$ and in particular $\mathbf{L}_{\boldsymbol{q}}^{(4) *}(21.3 s)$ increase. This indicates a nighttime increase in non Gaussianity (i.e., magnitude of $\mathbf{L}_{\boldsymbol{q}}^{(2)}$ ) that is driven both by purely asymmetric $\left(\mathbf{L}_{\boldsymbol{q}}^{(4) *}\right)$ and even order $\left(\mathbf{L}_{\boldsymbol{q}}^{(4)}\right)$ properties and characterized by stronger deviation from log-Normal (larger $\mathbf{L}_{\boldsymbol{q}}^{(4)}$ ). In [42], the non Gaussian behavior was reported to have the heaviest tails at scale $\approx 100 \mathrm{~s}$ during sleep, and the observed $\mathbf{L}_{\boldsymbol{q}}^{(2)}$ in healthy subjects is consistent with this observation. The non Gaussian distributions with non log-Normal-type heavy tails could be a consequence of the intermittent changes of sleep stages, as discussed above.

Such a circadian modulation of the non Gaussian properties of HRV is not present for $\mathrm{CHF}$ patients. $\mathrm{CHF}$ is characterized by autonomic imbalance including decreased parasympathetic activity and sympathetic hyperactivity. Thus, sympatheticdominant autonomic imbalance may weaken the circadian pattern of the HRV modulation.

4) Discriminative power of multiscale statistics of $H R V$ : We illustrate the discriminative power of the observed differences in multiscale distributions as indicators of mortality risk in CHF. In Fig. 10 (left), KMCS and MH statistics are plotted for $\mathbf{L}_{\boldsymbol{q}}^{(2)}(85.3 \mathrm{~s})$, which yielded most significant WRS p-value in the previous sections and is similar to the historical example [3] (there, computed for detrended increments). The threshold of the survival curves was chosen such as to maximize the $\mathrm{MH}$ $\log$ rank. The non Gaussian index $\mathbf{L}_{\boldsymbol{q}}^{(2)}(85.3)$ yields a $\mathrm{p}$-value of 0.0003 and the $\mathrm{MH} \log$ rank of 13.0 , which is consistent with the results reported in [3], and leads to sensitivity (specificity) of $66 \%(71 \%)$, respectively. Similar results are obtained for $\mathbf{L}_{\boldsymbol{q}}^{(2) *}(85.3), \mathbf{L}_{\boldsymbol{q}}^{(4)}(21.3)$ and $\mathbf{L}_{\boldsymbol{q}}^{(4) *}(21.3)$.

For comparison, KMCS and MH statistics for spectral and entropy based analyses (best results obtained for sampEN and apEN, reported in Fig. 11) are significant but substantially less predictive ( $\mathrm{p}$-value $>0.01, \mathrm{MH} \log$ rank $<7$, specificity $\leq 40 \%$; results for spectral analysis are worse, with $\mathrm{p}$-value $\geq$ $0.04, \mathrm{MH} \log$ rank $<4.5$, and not reported for space reason).

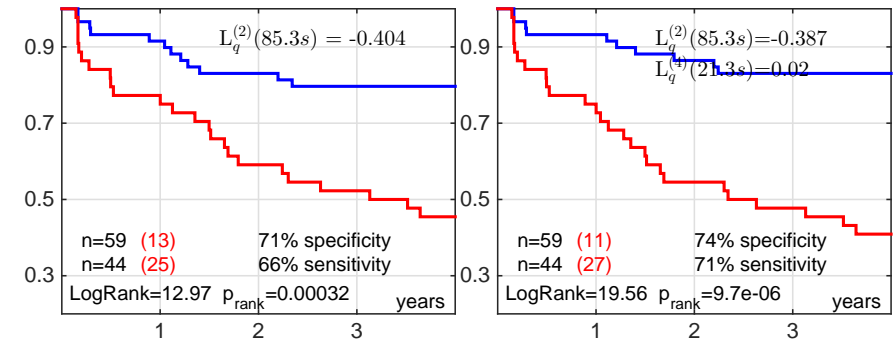

Fig. 10: KMCS for $\mathbf{L}_{\boldsymbol{q}}^{(2)}$ (left) and combined $\left(\mathbf{L}_{\boldsymbol{q}}^{(2)}, \mathbf{L}_{\boldsymbol{q}}^{(4)}\right)$ (right) with $\mathrm{MH} \log$ rank test.

Fig. 10 (right) further investigates the combined use of non Gaussian expansion indices and plots the KMCS and $\mathrm{MH}$ statistics when $\mathbf{L}_{\boldsymbol{q}}^{(2)}(85.3)$ and $\mathbf{L}_{\boldsymbol{q}}^{(4)}(21.3)$ are used together. It indicates that the combined predictive power of non Gaussian indices significantly improves predictive power, leading to $\mathrm{p}$ value smaller than $10^{-5}$, log rank of 19.5 , increasing sensitivity by $5 \%$ and specificity by $3 \%$, respectively. This suggests that the precise nature of non Gaussian is a discriminative feature for $\mathrm{CHF}$ mortality risk stratification and leads us to conclude that non Gaussian expansion indices are useful indicators of mortality risk in $\mathrm{CHF}$.

\section{CONCLUSIONS}

This work proposed the construction of novel, versatile non Gaussian multiscale representations for the use of a large continuum of scales without a priori assuming scalefree dynamics assumptions and the joint analysis of customizable ranges of (higher) statistical orders. The representations remain embedded in the recently proposed wavelet $p$-leader multifractal formalism and inherit from its theoretical grounding and practical robustness. These non Gaussian multiscale representations were used here for investigating the nonlinear dynamics of HRV for a database of 24 hour long HRV recordings from 90 healthy subjects and $108 \mathrm{CHF}$ patients. It enabled us to evidence the existence of short-term cascadetype multifractal mechanisms underlying HRV for both healthy and $\mathrm{CHF}$ subjects for time scales below 3 minutes. Further evidence was provided for the circadian evolution of this mechanism for the healthy group, while the constancy of multifractal parameters suggests a difference in the sympatheticparasympathetic balance for CHF patients. Moreover, several specific non Gaussian indices were constructed and individually evidenced to possess high discriminative abilities between $\mathrm{NS}$ and SV CHF patients for specific time scales $(21.3 \mathrm{~s}$ and $85.3 \mathrm{~s}$ ), substantially improving upon spectral, entropy and multifractal based indices. Future work will include studying the use of different combinations of higher order cumulants in the non Gaussian multiscale representation, and the precise assessment of the combined predictive power of non Gaussian indices for mortality risk in CHF patients.

\section{REFERENCES}

[1] T. Nakamura, K. Kiyono, H. Wendt, P. Abry, and Y. Yamamoto, "Multiscale analysis of intensive longitudinal biomedical signals and its clinical applications," Proc. IEEE, vol. 104, no. 2, pp. 242-261, 2016. 


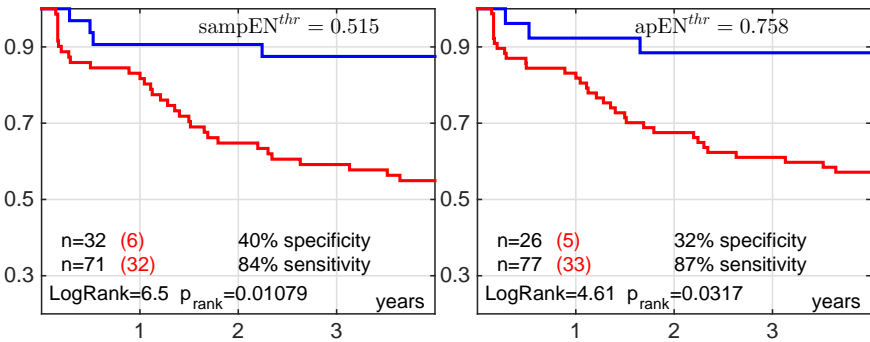

Fig. 11: KMCS for sampEN (left) and apEN (right).

[2] T. Makikallio, H. Huikuri, U. Hintze et al., "Fractal analysis and timeand frequency-domain measures of heart rate variability as predictors of mortality in patients with heart failure," Am. J. Cardiol., vol. 87, pp. 178-182, 2001.

[3] K. Kiyono, J. Hayano, E. Watanabe, Z. R. Struzik, and Y. Yamamoto, "Non-Gaussian heart rate as an independent predictor of mortality in patients with chronic heart failure," Heart Rhythm, vol. 5, pp. 261-268, 2008.

[4] M. D. Costa, A. L. Goldberger, and C.-K. Peng, "Multiscale entropy analysis of biological signals," Physical review E, vol. 71, no. 2, p. 021906, 2005.

[5] M. D. Costa, C.-K. Peng, and A. L. Goldberger, "Multiscale analysis of heart rate dynamics: entropy and time irreversibility measures," Cardiovascular Engineering, vol. 8, no. 2, pp. 88-93, 2008.

[6] P. C. Ivanov, L. A. N. Amaral, A. L. Goldberger, S. Havlin, M. G. Rosenblum, Z. R. Struzik, and H. E. Stanley, "Multifractality in human heart rate dynamics," Nature, vol. 399, pp. 461-465, 1999.

[7] C.-. K. Peng, S. Havlin, H. E. Stanley, and A. L. Goldberger, "Quantification of scaling exponents and crossover phenomena in nonstationary heartbeat time series," Chaos, vol. 5, pp. 82-87, 1995.

[8] K. Kiyono, Z. R. Struzik, N. Aoyagi, and Y. Yamamoto, "Multiscale probability density function analysis: Non-Gaussian and scale-invariant fluctuations of healthy human heart rate," IEEE Trans. Biomed. Eng. vol. 53, pp. 95-102, 2006.

[9] K. Kiyono, J. Hayano, S. Kwak, E. Watanabe, and Y. Yamamoto, "Non-gaussianity of low frequency heart rate variability and sympathetic activation: lack of increases in multiple system atrophy and parkinson disease," Front. Physiol., p. 89, 2012.

[10] J. Nolan and et al., "Prospective study of heart rate variability and mortality in chronic heart failure: Results of the united kingdom heart failure evaluation and assessment of risk trial," Circulation, vol. 98, pp. $1510-1516,1998$.

[11] D. P. Redfearn, "Non-gaussian index: Hope yet for the grail seekers?" Heart Rhythm, vol. 5, no. 2, pp. 269-270, 2008.

[12] S. Akselrod, D. Gordon, F. A. Ubel, D. C. Shannon, A. C. Berger, and R. J. Cohen, "Power spectrum analysis of heart rate fluctuation: a quantitative probe of beat-to-beat cardiovascular control," Science, vol. 213, no. 4504, pp. 220-222, 1981.

[13] B. Pomeranz, R. J. Macaulay, M. A. Caudill et al., "Assessment of autonomic function in humans by heart rate spectral analysis," Am. $J$. Physiol.-Heart Circul. Physiol., vol. 248, no. 1, pp. H151-H153, 1985.

[14] J. P. Saul, Y. Arai, R. D. Berger, L. S. Lilly, W. S. Colucci, and R. J. Cohen, "Assessment of autonomic regulation in chronic congestive heart failure by heart rate spectral analysis," Am. J. Cardiol., vol. 61, no. 15, pp. 1292-1299, 1988.

[15] D. Bansal, M. Khan, and A. Salhan, "A review of measurement and analysis of heart rate variability," Proc. IEEE Int. Conf. Comput. Autom. Eng. (ICCAE), pp. 243-246, 2009.

[16] U. R. Acharya, K. P. Joseph, N. Kannathal, C. M. Lim, and J. S. Suri, "Heart rate variability: a review," Med. Biol. Eng. Comput., vol. 44, no. 12, pp. 1031-1051, 2006.

[17] S. Jasson, C. Médigue, P. Maison-Blanche et al., "Instant power spectrum analysis of heart rate variability during orthostatic tilt using a time/frequency-domain method," Circulation, vol. 96, no. 10, pp. 3521-3526, 1997.

[18] M. Kobayashi and T. Musha, "I/f Fluctuation of heartbeat period," IEEE Trans. Biomed. Eng., vol. BME-29, pp. 456-457, 1982.

[19] C. K. Peng, J. Mietus, J. M. Hausdorff, S. Havlin, H. E. Stanley, and A. L. Goldberger, "Long-range autocorrelations and non-Gaussian behavior of the heartbeat," Phys. Rev. Lett., vol. 70, pp. 1343-1346, 1993.
[20] Y. Yamamoto and R. L. Hughson, "On the fractal nature of heart rate variability in humans: effects of data length and $\beta$-adrenergic blockade," Am. J. Physiol., vol. 266, no. Regulatory Integrative Comp. Physiol. 35, pp. R40-R49, 1994.

[21] L. A. N. Amaral, P. C. Ivanov, N. Aoyagi et al., "Behavioral-independent features of complex heartbeat dynamics," Phys. Rev. Lett., vol. 86, pp. 6026-6029, 2001

[22] M. Doret, J. Spilka, V. Chudáček, P. Gonçalves, and P. Abry, "Fractal analysis and hurst parameter for intrapartum fetal heart rate variability analysis: A versatile alternative to frequency bands and lf/hf ratio," PloS one, vol. 10, no. 8, p. e0136661, 2015.

[23] M. Doret, H. Helgason, P. Abry, P. Gonçalvès, C. Gharib, and P. Gaucherand, "Multifractal analysis of fetal heart rate variability in fetuses with and without severe acidosis during labor," Am. J. Perinatol., vol. 28, no. 4, pp. 259-266, 2011.

[24] V. Chudácek, J. Andén, S. Mallat, P. Abry, and M. Doret, "Scattering transform for intrapartum fetal heart rate variability fractal analysis: A case-control study," IEEE Trans. Biomed. Eng., vol. 61, no. 4, pp. 11001108, 2014.

[25] K. Kiyono, Z. R. Struzik, N. Aoyagi, S. Sakata, J. Hayano, and Y. Yamamoto, "Critical scale-invariance in healthy human heart rate," Phys. Rev. Lett., vol. 93, p. 178103, 2004.

[26] S. Jaffard, C. Melot, R. Leonarduzzi, H. Wendt, P. Abry, S. G. Roux, and M. E. Torres, "p-exponent and p-leaders, part i: Negative pointwise regularity." Physica A, vol. 448, pp. 300-318, 2016.

[27] R. Leonarduzzi, H. Wendt, P. Abry, S. Jaffard, C. Melot, S. G. Roux, and M. E. Torres, "p-exponent and p-leaders, part ii: Multifractal analysis. relations to detrended fluctuation analysis." Physica A, vol. 448, pp. 319-339, 2016.

[28] K. Kiyono, "Establishing a direct connection between detrended fluctuation analysis and Fourier analysis," Physical Review E, vol. 92, no. 4, p. 042925, 2015.

[29] K. Kiyono and Y. Tsujimoto, "Nonlinear filtering properties of detrended fluctuation analysis," Physica A: Statistical Mechanics and its Applications, vol. 462, pp. 807-815, 2016.

[30] S. Mallat, A Wavelet Tour of Signal Processing. San Diego, CA Academic Press, 1998.

[31] B. Castaing, Y. Gagne, and M. Marchand, "Log-similarity for turbulent flows," Physica D, vol. 68, pp. 387-400, 1993.

[32] H. Wendt, P. Abry, and S. Jaffard, "Bootstrap for empirical multifractal analysis," IEEE Signal Proc. Mag., vol. 24, no. 4, pp. 38-48, 2007.

[33] S. Jaffard, "Wavelet techniques in multifractal analysis," in Fractal Geometry and Applications: A Jubilee of Benoît Mandelbrot, M. Lapidus et M. van Frankenhuijsen Eds., Proceedings of Symposia in Pure Mathematics, vol. 72(2). AMS, 2004, pp. 91-152.

[34] S. Jaffard, P. Abry, and H. Wendt, Irregularities and Scaling in Signal and Image Processing: Multifractal Analysis. Singapore: World scientific publishing, 2015, pp. 31-116.

[35] J. Muzy, E. Bacry, and A. Arneodo, "The multifractal formalism revisited with wavelets," Int. J. of Bifurcation and Chaos, vol. 4, pp. 245-302, 1994.

[36] R. Lopes and N. Betrouni, "Fractal and multifractal analysis: A review," Medical Image analyis, vol. 13: 634-49, 2009.

[37] B. Mandelbrot and J. van Ness, "Fractional Brownian motion, fractional noises and applications," SIAM Reviews, vol. 10, pp. 422-437, 1968.

[38] E. Bacry, J. Delour, and J. F. Muzy, "Multifractal random walk," Phys. Rev. E, vol. 64, p. 026103, 2001.

[39] B. Mandelbrot, "Limit lognormal multifractal measures," in Frontiers of Physics, Proc. Landau Memorial Conf., Tel Aviv, 1988, E. Gotsman, Y. Ne'eman, and A. Voronel, Eds. Pergamon Press, 1990, pp. 309-340.

[40] R. Riedi, "Multifractal processes," in Theory and applications of long range dependence, P. Doukhan, G. Oppenheim, and M. Taqqu, Eds. Birkhäuser, 2003, pp. 625-717.

[41] A. Arneodo, C. Baudet, F. Belin et al., "Structure functions in turbulence, in various flow configurations, at Reynolds number between 30 and 5000, using extended self-similarity," EPL (Europhysics Letters), vol. 34, no. 6, p. 411, 1996.

[42] K. Kiyono, Z. R. Struzik, N. Aoyagi, F. Togo, and Y. Yamamoto, "Phase transition in healthy human heart rate," Phys. Rev. Lett., vol. 95, p. 058101, 2005.

[43] B. Mandelbrot, "Intermittent turbulence in self-similar cascades: divergence of high moments and dimension of the carrier," J. Fluid Mech., vol. 62, pp. 331-358, 1974.

[44] R. Furlan, S. Guzzetti, W. Crivellaro et al., "Continuous 24-hour assessment of the neural regulation of systemic arterial pressure and rr variabilities in ambulant subjects." Circulation, vol. 81, no. 2, pp. $537-547,1990$ 